\title{
VARIEDADES DE CANA-DE-AÇÚCAR SUBMETIDAS À IRRIGAÇÃO SUPLEMENTAR NO CERRADO GOIANO
}

\section{PATRICK F. CAMPOS ${ }^{1}$, JOSÉ ALVES JÚNIOR ${ }^{2}$, DERBLAI CASAROLI ${ }^{3}$, PATRICIA R. FONTOURA $^{4}$, ADÃO W. P. EVANGELISTA ${ }^{5}$}

\begin{abstract}
RESUMO: O Estado de Goiás é o segundo maior produtor de cana-de-açúcar do Brasil. Entretanto, ainda apresenta baixa produtividade, devido principalmente à falta de variedades adaptadas a esta região e ao déficit hídrico típico. Assim, objetivou-se avaliar o desempenho de dezesseis variedades de cana-de-açúcar submetidas à irrigação suplementar no Cerrado. O experimento foi conduzido em Goianésia-GO, em um Latossolo Vermelho-Amarelo Distrófico, de textura argilosa de abril de 2011 a maio de 2012. O delineamento foi em blocos casualizados (Bifatorial 16x5), com 16 tratamentos (variedades): CTC2, CTC4, CTC9, CTC11, CTC15, CTC18, IAC87-3396, IAC91-1099, IACSP943046, IACSP94-2094, IACSP94-2101, IACSP95-5000, RB857515, RB92579, RB966928 e SP860042; e cinco tempos de avaliação: 113; 145; 174; 203 e 285 dias após o plantio (DAP), com quatro repetições. A irrigação suplementar, por aspersão, correspondeu a uma reposição de $50 \%$ da ETc. Os resultados, de cana- planta, apresentaram diferenças significativas entre as variedades avaliadas, destacando-se as variedades CTC9, CTC11, IAC87-3396, IAC91-1099 e SP86-0042, em termos de produtividade e de rendimento industrial. Assim, estas variedades podem ser recomendadas para 0 cultivo no Cerrado, em regime de irrigação suplementar.
\end{abstract}

PALAVRAS-CHAVE: Saccharum officinarum, déficit hídrico, manejo da irrigação, aspersão.

\section{VARIETIES OF SUGARCANE TO SUPPLEMENTAL IRRIGATION IN BRAZILIAN SAVANNA}

\begin{abstract}
The state of Goias is the second largest producer of sugar cane in Brazil. However, it still has low productivity, mainly due to the lack of varieties adapted to the region and the typical water deficit. The experiment aimed to evaluate the performance of sixteen sugarcane varieties submitted to supplementary irrigation in the "Cerrado" (Brazilian Savanna).The experiment was carried out in Goianesia-GO (Brazil), in a Red yellow dystrophic latosol (Oxisol), from April 2011 to May 2012. Randomized blocks design was used, with bifatorials treatments, in sixteen sugar cane varieties: CTC2, CTC4, CTC9, CTC11, CTC15, CTC18, IAC87-3396, IAC91-1099, IACSP943046, IACSP94-2094, IACSP94-2101, IACSP95-5000, RB857515, RB92579, RB966928 and SP86-0042; and five evaluation time: 113, 145, 174, 203 and 285 days after plantation (DAP). The supplementary irrigation was of $50 \%$ water requirement using a sprinkler system. Varieties performance was evaluated through: tillering, growth, productivity and performance of industrial plants. The results showed significant differences between the varieties. The best results were obtained for varieties CTC9, CTC11, IAC87-3396, IAC91-1099 and SP86-0042, to productivity and industrial performance, which can be recommended in savannah cultivation with supplementary irrigation (50\%).
\end{abstract}

KEYWORDS: Saccharum officinarum, water stress, irrigation management, sprinkler.

\footnotetext{
${ }^{1}$ Engo Agrônomo, Mestre em Agronomia - EA/UFG, Pesquisador da Usina Jales Machado, Goianésia - GO, Fone: (62) 84700137,pfcagro@gmail.com

${ }^{2}$ Eng ${ }^{\circ}$ Agrônomo, Prof. Doutor, Escola de Agronomia (EA), Universidade Federal de Goiás (UFG), Goiânia - GO, josealvesufg@yahoo.com.br

${ }^{3}$ Eng $^{\circ}$ Agrônomo, Prof. Doutor, Escola de Agronomia (EA), Universidade Federal de Goiás (UFG), Goiânia - GO, derblaicasaroli@yahoo.com.br

${ }^{4}$ Eng $^{\circ}$ Agrônomo, Mestre em Agronomia - EA/UFG, Pesquisador da Usina Jales Machado, Goianésia - GO, Fone: (62) 84700137, patricia.rezende@jallesmachado.com.br

${ }^{5}$ Eng ${ }^{0}$ Agrícola, Prof. Doutor, EA-UFG, Goiânia - GO, awpego@bol.com.br

Recebido pelo Conselho Editorial em: 11-7-2013
}

Aprovado pelo Conselho Editorial em: 05-6-2014 


\section{INTRODUÇÃO}

A cana-de-açúcar é considerada a cultura agrícola mais produzida no mundo, sendo colhidas mais de um bilhão de toneladas por ano (CONAB, 2012). Ainda, a cana é responsável por 70\% da produção do açúcar mundial, superando a beterraba, sendo utilizada também como fonte de energia. Por isso, o cultivo da cana-de-açúcar tem-se expandido, sobretudo em regiões de clima tropical e subtropical. Tendo em vista que os períodos de seca reduzem significativamente a produtividade da cana, o desenvolvimento de variedades adaptadas a esta condição, possivelmente, será um fator determinante para o aumento da produção e a ampliação de novas áreas de cultivo (HENRY, 2010).

A Índia foi, há trinta anos, o maior produtor de cana. Atualmente, o Brasil é o país com a maior produção, representando 23\% da produção mundial. Para a safra de 2012/2013, estima-se uma área destinada ao setor sucroalcooleiro de 8,52 milhões de hectares. A produção brasileira concentra-se no Estado de São Paulo (maior produtor), com uma área plantada de 4,4 milhões ha (51,87\%) e uma produtividade média de 73,1 $\mathrm{Mg} \mathrm{ha}^{-1}$, seguido por Goiás com 725.910 ha (8,52\%) e 70,7 $\mathrm{Mg} \mathrm{ha}^{-1}$ de produtividade e Minas Gerais com 721.850 ha $(8,47 \%)$ e produtividade de 74,2 $\mathrm{Mg}$ ha $^{-1}$ (CONAB, 2012).

O Vale do São Patrício é uma importante região produtora de cana em Goiás, com destaque para o município de Goianésia. Este município tem a segunda maior área plantada do Estado, com 45.000 ha, inferior apenas a Quirinópolis (58.500 ha) e Turvelândia (45.300ha) (CANASAT, 2013). Esses três municípios também apresentam as maiores produções: Quirinópolis $(4.121 .700 \mathrm{Mg}$ e 69,3 $\mathrm{Mg} \mathrm{ha}^{-1}$ de produtividade), Turvelândia (3.380.000 Mg e 70,2 $\mathrm{Mg}^{-1}$ de produtividade) e Goianésia (3.279.100 Mg e 60,4 $\mathrm{Mg} \mathrm{ha}^{-1}$ de produtividade) (CONAB, 2012). Tendo em vista que a cana necessita de uma precipitação acumulada acima de $1.000 \mathrm{~mm} \mathrm{ano}^{-1}$ (MARIN\&NASSIF, 2013), a disponibilidade hídrica nessa região pode ser considerada o principal fator da variabilidade produtiva, ciclo após ciclo da cultura, sendo este um aspecto limitante para o aumento da produtividade na região do Vale do São Patrício, pois esta, apesar de apresentar uma precipitação anual média de $1.540 \mathrm{~mm}$, apresenta déficit hídrico de até cinco meses por ano. Isto acarreta produtividades baixas, em torno de 60,4 $\mathrm{Mg} \mathrm{ha}^{-1}$, aquém de importantes regiões paulistas, com 85 $\mathrm{Mg} \mathrm{ha}^{-1}$ (KOHLHEPP, 2010).

Para obter rendimentos máximos em colmos, a cultura deve contar com água no solo e temperatura do ar adequada durante todos os estádios vegetativos. O crescimento e o desenvolvimento da cana são diretamente proporcionais à água transpirada, pois existe uma relação linear entre a evapotranspiração da cana-de-açúcar e sua produtividade (LEAL, 2012). Os danos causados pelo déficit hídrico à expansão foliar e à produtividade desta cultura dependem da intensidade e da duração deste período, da fase de desenvolvimento da cultura e da variedade cultivada (MACHADO et al., 2009).

A irrigação é uma prática benéfica para agricultura, pois proporciona condições adequadas de água no solo durante o ciclo da cultura, desde que conduzida com técnica e sistema adequados. Aumentos na produtividade da cana-de-açúcar, quando irrigada de forma plena, são observados extensamente na literatura (FARIAS et al., 2008; DALRI \&CRUZ, 2008). Entretanto, este manejo não é comum para a cana-de-açúcar, por esta apresentar áreas extensivas de cultivo, requerendo elevados investimentos. O uso de variedades resistentes ou tolerantes seria outra alternativa para minimizar as quebras de produtividade associadas ao déficit hídrico.

A cana-de-açúcar é a cultura agrícola com maior área irrigada no Brasil, pois dos 5,4 milhões de hectares irrigados no País, 1,7 milhão é com a cultura da cana, podendo chegar a irrigar 29,5 milhões de hectares (ANA, 2012). O objetivo deste trabalho foi avaliar o desempenho de variedades de cana-de-açúcar, submetidas à irrigação suplementar no Cerrado em Goiás. 


\section{MATERIAL E MÉTODOS}

O experimento foi instalado em Latossolo Vermelho-Amarelo distrófico (EMBRAPA, 2006), na Usina Jales Machado (senso varietal 2013 com 10\% das áreas cultivadas com a variedade RB867515, 9\% com CTC-04, 7\% com IAC91-1099, 7\% com CTC-15, 5\% com IAC87-3396, 5\% com CTC-18, 4\% com SP84-1431, 3\% com SP86-0042, 3\% com SP79-1011, 3\% com CTC-02, 2\% com IACSP95-5000, 2\% com IACSP94-2101, 1\% com CTC-09, 1\% com CT94-3166, 1\% com SP835073, 1\% com SP83-2847, 35\% com outras), no município de Goianésia-GO (coordenadas: $15^{\circ} 12^{\prime}$ S; $48^{\circ} 59^{\prime} \mathrm{W}$; e 580 m de altitude).

Segundo Köppen, o clima é classificado como um Aw, denominado tropical de savana (inverno seco e verão chuvoso). A média pluviométrica anual é de $1.540 \mathrm{~mm}$, com período de déficit hídrico bem definido, entre maio e outubro (Figura 1).

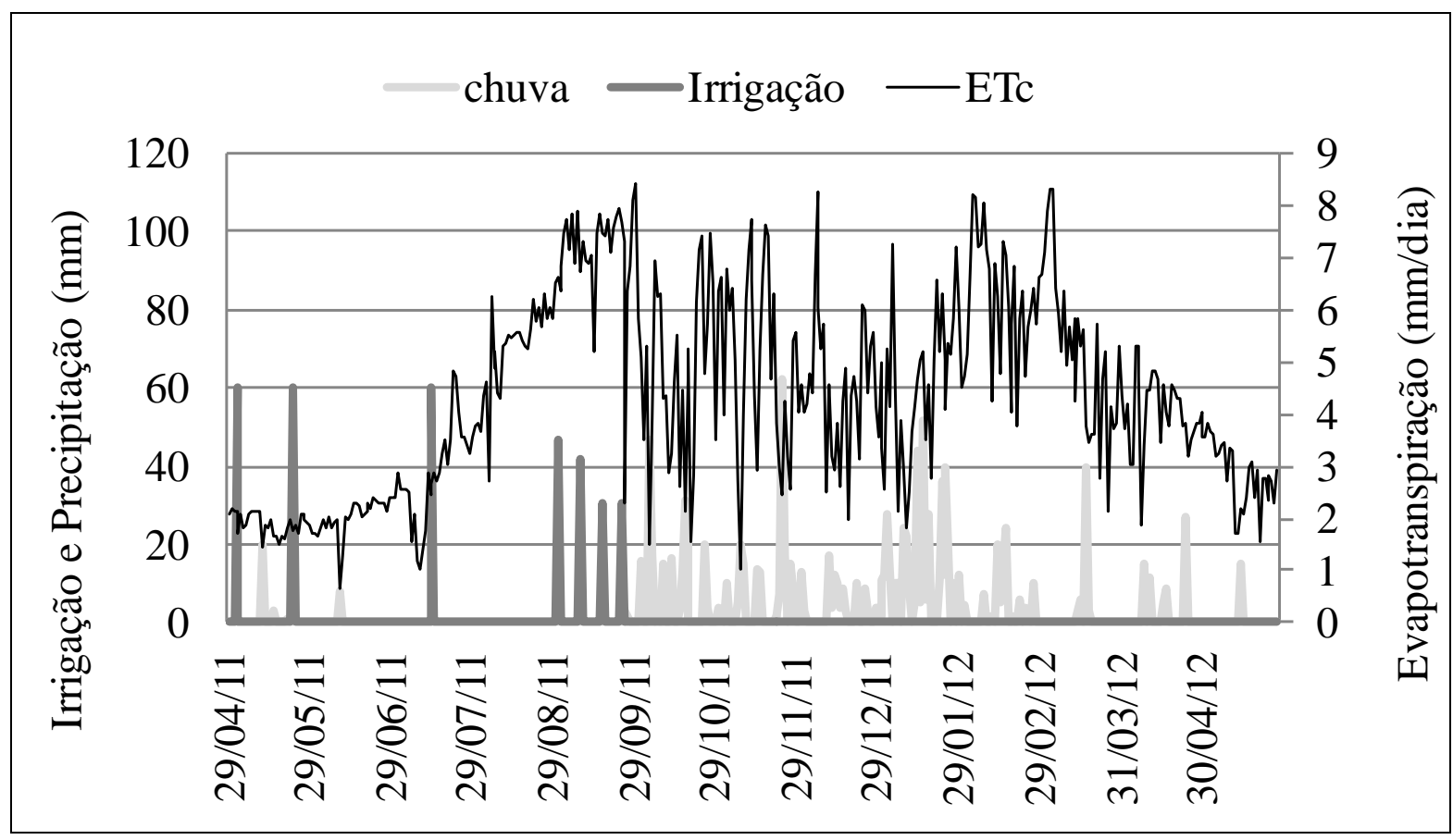

FIGURA 1. Balanço hídrico da cana-de-açúcar, com totais mensais de chuva, evapotranspiração da cultura e irrigações, Goianésia-GO (abril de 2011 a maio 2012). Water balance of sugar cane crop, total monthly of rainfall, crop evapotranspiration and irrigation, Goianésia-GO (April 2011 to May 2012).

Anterior à instalação do experimento, a área continha pastagem degradada, com predomínio de capim braquiária (Brachiaria decumbens). Seis meses antes do preparo da área, foram coletadas amostras deformadas de solo, para análise química, nas camadas de 0,0-0,25 m e 0,25-0,50 m, e amostras indeformadas, para as análises físico-hídricas do solo, nas camadas de 0,0-0,30 m e 0,300,60 m. Para a correção do solo, utilizou-se de calcário dolomítico, elevando a saturação de bases a $50 \%$, aplicaram-se, ainda, gesso agrícola $\left(2.250 \mathrm{~kg} \mathrm{ha}^{-1}\right)$ e $\mathrm{P}_{2} \mathrm{O}_{5}\left(100 \mathrm{~kg} \mathrm{ha}^{-1}\right)$, na forma de fosfato natural.

O preparo do solo foi baseado em uma gradagem pesada, para a incorporação de corretivos. Posteriormente, efetuou-se uma gradagem intermediária para a incorporação do fosfato natural e a quebra de torrões. E por fim, para o nivelamento do terreno, uma gradagem leve antes do plantio.

No fundo do sulco de plantio, a 0,35 m de profundidade, distribuíram-se $115 \mathrm{~kg}$ de $\mathrm{P}_{2} \mathrm{O}_{5} \mathrm{ha}^{-1}$ (superfosfato triplo). O plantio da cana foi realizado manualmente, no dia 29-04-2011, contendo os toletes três gemas vegetativas. Após, cobriram-se os sulcos e aplicou-se o inseticida fipronil800 WG (0,050 kg ha ${ }^{-1}$ p.c.) para prevenção de cupins. Devido à época de plantio, houve a necessidade de aplicar uma lâmina de irrigação de 40 mm, para estimular a brotação da cana. 
O delineamento experimental utilizado foi o de blocos ao acaso, em que os tratamentos formavam um esquema bifatorial 16x5, sendo, respectivamente, 16 variedades de cana-de-açúcar: CTC2, CTC4, CTC9, CTC11, CTC15, CTC18, IAC87-3396, IAC91-1099, IACSP94-3046, IACSP94-2094, IACSP94-2101, IACSP95-5000, RB857515, RB92579, RB966928 e SP86-0042; e cinco tempos de avaliação: 113; 145; e 174; 203 e 285 dias após o plantio (DAP), com quatro repetições. As unidades experimentais foram formadas por parcelas de quatro linhas, espaçadas a $1,5 \mathrm{~m}$, e $15 \mathrm{~m}$ de comprimento $\left(90 \mathrm{~m}^{2}\right)$.

O manejo de irrigação foi realizado determinando a demanda hídrica da cana-de-açúcar, utilizando-se de coeficientes da cultura (Kc) igual a 0,5; 0,8; 1,25 e 0,8, para os respectivos estádios fenológicos da cultura: germinação (30 dias), desenvolvimento (140 dias), desenvolvimento pleno (145 dias) e maturação (81 dias) (DALRI \& CRUZ, 2008). Para a estimativa da evapotranspiração de referência (ETo), utilizou-se do modelo de Penman-Monteith-FAO. Os dados (Figura 1) foram obtidos na estação meteorológica automática do INMET, localizada na usina Jalles Machado, próximo da área experimental $(3 \mathrm{~km})$. A lâmina bruta de irrigação foi calculada por meio de balanço hídrico, em que as entradas de água foram a irrigação e a precipitação pluvial efetiva, e as saídas, a evapotranspiração da cultura (ETc) em cada estádio fenológico, considerando uma eficiência de aplicação de 83\%. A irrigação suplementar supriu 50\% da necessidade hídrica da cultura. Desta forma, durante o ciclo, aplicou-se uma lâmina bruta de irrigação de 369,81 mm, que, somada à chuva (1.209,03 mm), totalizou uma lâmina de 1.578,84 mm, no ciclo (Figura 1).

Foi utilizado para irrigação o equipamento autopropelido tipo carretel enrolador modelo Turbomaq 140/GSV/350-4RII, implementado com barra irrigadora modelo 48/54 fabricada pela empresa "IrrigaBrasil”, com faixa de aplicação de 54 metros, com vão livre máximo da barra até o solo variando de $1,00 \mathrm{~m}$ a 4,00 m. Foi utilizado o aspersor tipo Spray LDN® ${ }^{\circledR}$ com bocais Senninger \#21 (vazão 109,90 L h${ }^{-1}$ e pressão de serviço de 36 m.c.a.) e regulador de pressão Senninger de 20 psi.

Alguns tratos culturais foram realizados durante o ciclo da cana: sistematização das entrelinhas com a linha da cana; adubação de cobertura (05-00-12 + 0,3\% B + 0,3\% Zn na dose de $1.200 \mathrm{~kg} \mathrm{ha}^{-1}$ ); controle de plantas daninhas em pré-emergência.

O estudo foi conduzido durante o ciclo da cana-planta, colhida aos 396 dias após o plantio (29-05-2012), utilizando-se da colheita mecanizada (colhedora com esteira rodante - John Deere, modelo 3510 e caminhão transbordo com dispositivo de célula de carga).

Realizaram-se as seguintes avaliações de desempenho: i) número de perfilhos: contabilizou-se o número de perfilhos por metro linear, nas duas linhas centrais de cada parcela. Esta avaliação foi feita aos 113; 145; 174 e 203 dias após o plantio (DAP); ii) comprimento de colmos: avaliou-se a altura média do dossel de cada parcela (série temporal: 113; 145 e 174 DAP). E aos 203 DAP considerou-se a distância do solo até a primeira folha dewlep visível, e para amostragem, no período 285 DAP, mediu-se o comprimento de colmo de 10 canas de cada parcela (desconsiderado o palmito); iii) diâmetro de colmo: medido no terço médio inferior, com o auxílio de um paquímetro, em 10 colmos por parcela, aos 285 DAP; iv) massa de colmo: a fitomassa verde de 10 colmos foi determinada por meio de balança suspensa digital, do tipo gancho, aos 285 DAP. E aos 396 DAP (momento da colheita), utilizando 10 colmos por parcela, realizaram-se as seguintes avaliações tecnológicas: i) massa do bagaço úmido; ii) teor de sólidos solúveis do caldo ( ${ }^{\circ}$ BRIX, \%); iii) teor de sacarose (POL, \%); iv) pureza do caldo (quantificação); v) fibra (quantificação); vi) umidade (quantificação); vii) açúcares totais recuperáveis (ATR); viii) tonelada de POL por hectare - TPH: obtida pela multiplicação do valor de POL pela produtividade real.

Os dados foram submetidos à análise de variância, e as médias, comparadas pelo teste de Tukey, em nível de 5\% de probabilidade de erro. Para isto, utilizou-se o pacote estatístico Assistat (7.6 beta). 


\section{RESULTADOS E DISCUSSÃO}

\section{Crescimento de plantas}

Para o crescimento de plantas, houve interação significativa entre os dois fatores analisados, variedades e estádios de desenvolvimento ( $p>0,05)$, com destaque para CTC9, IAC87-3396 e RB966928, por apresentarem maior comprimento de colmos e maior número de perfilhos ao longo do ciclo. As variedades CTC11, CTC15, RB867515 e SP86-0042 destacaram-se em comprimento de colmos; entretanto, não apresentaram destaque em número de perfilhos, e o contrário ocorreu com as variedades CTC2 e IAC91-1099. Os valores mínimos e máximos de comprimento de colmos, das 16 variedades, aos 113; 145; 174; 203 e 285 dias após o plantio (DAP), foram 0,75 a 1,10; 0,92 a $1,45 \mathrm{~m} ; 1,05$ a $1,50 \mathrm{~m} ; 1,57$ a 2,26 m, e 1,99 a 2,71 m, respectivamente. A redução observada nos valores, aos 203 e 285 DAP (Tabela 1), pode ter ocorrido devido à mudança na metodologia para avaliar esta variável. Além disto, aos 285 DAP, as variedades CTC9 e IAC87-3396 também obtiveram destaque para a variável diâmetro médio de colmo, os quais variaram entre 32,15 e 25,23 mm. Os menores e maiores valores observados de número de perfilhos, para os quatro tempos de avaliação (113; 145; 174 e 203 DAP) foram: 9,58 e 21,10 perf. $\mathrm{m}^{-1} ; 14,03$ e 20,62 perf. $\mathrm{m}^{-1} ; 14,58$ e 21,10 perf. $\mathrm{m}^{-1} ; 12,35$ e 16,47 perf. $\mathrm{m}^{-1}$; respectivamente.

Em geral, todas as variedades estudadas, ao longo do ciclo, apresentaram diferença estatística na variável comprimento de colmo. Para esta variável, sete variedades (CTC9, CTC11, CTC15, IAC87-3396, RB867515, RB966928 e SP86-0042) obtiveram as maiores médias, nos cinco tempos de avaliação. Desta forma, pode-se inferir que o déficit hídrico não prejudicou o crescimento dos colmos destas variedades (Tabela 1), com crescimento destacado desde o início do ciclo. Este crescimento contínuo e uniforme pode contribuir para o aumento da eficiência fotossintética das plantas e para o manejo de plantas daninhas, sobretudo devido ao fechamento rápido das entrelinhas e à cobertura homogênea do solo (BENNET et al. (2011). SILVA et al. (2008) ressaltam que as variações de altura de plantas, ou comprimento de colmos de cana-de-açúcar, são indicativos de tolerância ao déficit hídrico. Desta forma, estas variedades mostraram-se responsivas à irrigação suplementar, sendo esta uma prática relevante para manter o processo produtivo mesmo em condições de baixa disponibilidade hídrica às plantas.

O déficit hídrico no início do ciclo (abril-outubro), a insuficiência da irrigação suplementar (com reposição de apenas 50\% da ETc) e o padrão de crescimento das variedades (CTC4, CTC18, IACSP93-3096, RB92579 e RB92579) avaliadas podem ter ocasionado a obtenção de menores médias de comprimento de colmo. Pois, a falta de água nos tecidos vegetais impacta os processos relacionados à turgescência, fechamento estomático, fotossíntese, respiração e, consequentemente, os padrões de crescimento e desenvolvimento vegetal (SHABANI et al., 2013). MACHADO et al. (2009) detectaram alterações na condutância estomática das variedades IACSP94-2094 e IACSP962042 durante todas as fases fenológicas da cultura, implicando a redução do porte da planta, devido a uma redução na atividade fotossintética. GONÇALVES et al. (2010) observaram que a condutância estomática, a transpiração foliar, a fotossíntese líquida e a eficiência de uso da água de produção das variedades IACSP791011, RB72454, RB98710 e RB92579 foram prejudicadas pela condição de déficit hídrico. As variedades IACSP94-2101 e CTC2 apresentaram, aos 285 DAP, a altura de 2,08 e 2,33 metros, respectivamente. Estes resultados representam a redução de quase $10 \%$ em relação aos valores observados por SANTIN et al. (2009), em Jaboticabal-SP. No entanto, essas diferenças podem estar associadas ao período de amostragem, que ocorreu na ocasião da colheita, representando a diferença de 110 dias. 
TABELA 1. Comprimento de colmos (Cc, m) e número de perfilhos (№ perfilhos), nos períodos de 113; 145; 174; 203 e 285 DAP. Length of stems (Cc in meter), and tillering ( $\mathbf{N}^{\mathbf{0}}$ perfilhos), in 113, 145, 174, 203 and 285 DAP (Day after planting).

\begin{tabular}{|c|c|c|c|c|c|c|c|c|c|c|}
\hline \multirow[t]{2}{*}{ Tratamentos } & \multicolumn{2}{|c|}{113 DAP } & \multicolumn{2}{|c|}{145 DAP } & \multicolumn{2}{|c|}{174 DAP } & \multicolumn{2}{|c|}{203 DAP } & \multicolumn{2}{|c|}{285 DAP } \\
\hline & $\mathrm{Cc}$ & $\begin{array}{c}\text { № } \\
\text { perfilhos }\end{array}$ & $\mathrm{Cc}$ & $\begin{array}{c}\text { № } \\
\text { Perfilhos }\end{array}$ & $\mathrm{Cc}$ & $\begin{array}{c}\text { № } \\
\text { perfilhos }\end{array}$ & $\mathrm{Cc}$ & № perfilhos & $\mathrm{Cc}$ & № perfilhos \\
\hline $\mathrm{CTC2}$ & $1,08 \mathbf{a b C}$ & $18,54 \mathbf{a b c A}$ & $1,35 \mathbf{a b B}$ & $19,09 \mathbf{a b A}$ & $2,16 \mathbf{a b c A}$ & $19,14 \mathbf{a b A}$ & $1,35 \mathbf{a b c d B}$ & $16,41 \mathbf{a A}$ & 2,33 bcdef $A$ & - \\
\hline CTC4 & $0,75 \mathbf{d C}$ & 13,21 cdefC & $0,94 \mathrm{eB}$ & $18,39 \mathbf{a b c A B}$ & $1,64 \mathrm{efA}$ & $21,11 \mathbf{a A}$ & 1,24 cdeB & $15,88 \mathbf{a B C}$ & $2,46 \mathbf{a b c A}$ & - \\
\hline CTC9 & $1,09 \mathbf{a b C}$ & $21,10 \mathbf{a A}$ & $1,41 \mathbf{a b B}$ & $18,74 \mathbf{a b c A B}$ & 2,11 abcdA & $19,81 \mathbf{a b A}$ & $1,50 \mathrm{aB}$ & $14,70 \mathrm{aB}$ & $2,71 \mathbf{a A}$ & - \\
\hline CTCll & $1,10 \mathbf{a C}$ & 15,24 bcdefA & $1,45 \mathrm{aB}$ & $18,29 \mathbf{a b c A}$ & $2,26 \mathbf{a A}$ & $16,86 \mathbf{a b A}$ & $1,40 \mathbf{a b c B}$ & $14,54 \mathbf{a A}$ & 2,44 abcdA & - \\
\hline CTCl5 & $0,93 \mathbf{a b c d C}$ & 14,54 bcdefA & $1,18 \mathbf{b c d B}$ & $14,85 \mathbf{a b c A}$ & 1,89 abcdefA & $18,53 \mathbf{a b A}$ & $1,41 \mathbf{a b c B}$ & $15,85 \mathbf{a A}$ & $2,49 \mathbf{a b c A}$ & - \\
\hline CTC18 & $0,84 \mathbf{c d C}$ & $9,58 \mathrm{fB}$ & $1,07 \mathrm{deB}$ & $14,03 \mathbf{b c A}$ & 1,81 bcdef $A$ & $14,58 \mathbf{b A}$ & 1,20 cdeB & $12,35 \mathbf{a A B}$ & 2,24 cdef $A$ & - \\
\hline IAC87-3396 & $1,04 \mathbf{a b c C}$ & $18,24 \mathbf{a b c d} \mathrm{A}$ & $1,28 \mathbf{a b c d B}$ & $16,20 \mathbf{a b c A B}$ & 1,93 abcdefA & $18,83 \mathbf{a b A}$ & $1,36 \mathbf{a b c d B}$ & $13,71 \mathbf{a B}$ & 2,39abcdeA & - \\
\hline LAC91-1099 & $0,94 \mathbf{a b c d C}$ & $19,62 \mathbf{a b A}$ & $1,22 \mathbf{a b c d B}$ & $17,88 \mathbf{a b c A}$ & 1,76 cdef $A$ & $20,26 \mathbf{a b A}$ & $1,19 \mathrm{deB}$ & $16,47 \mathbf{a A}$ & 2,11 defA & - \\
\hline LACSP93-3096 & $0,85 \mathbf{c d C}$ & 15,15 bcdef $A$ & $1,04 \mathrm{deB}$ & 16,75 abcA & $1,57 \mathbf{f A}$ & $18,79 \mathbf{a b A}$ & $1,05 \mathbf{e B}$ & $15,25 \mathbf{a A}$ & $1,99 \mathbf{f A}$ & - \\
\hline LACSP94-2094 & $0,88 \mathbf{b c d C}$ & $12,28 \mathbf{e f A}$ & 1,09 cdeB & $14,21 \mathbf{b c A}$ & 1,71 defA & $16,31 \mathbf{a b A}$ & $1,23 \mathbf{c d e B}$ & $16,05 \mathbf{a A}$ & 2,11 defA & - \\
\hline LACSP94-2101 & $0,99 \mathbf{a b c C}$ & $12,91 \mathbf{c d e f B}$ & $1,25 \mathbf{a b c d B}$ & $14,68 \mathbf{b c A B}$ & 2,00 abcdeA & $17,49 \mathbf{a b A}$ & $1,21 \mathbf{c d e B}$ & $14,62 \mathrm{aAB}$ & $2,08 \mathbf{e f A}$ & - \\
\hline LACSP95-5000 & $1,00 \mathbf{a b c C}$ & 14,58 bcdefA & $1,25 \mathbf{a b c d B}$ & $15,50 \mathbf{a b c A}$ & 1,90 abcdef $A$ & $16,39 \mathbf{a b A}$ & $1,26 \mathbf{b c d B}$ & $15,61 \mathbf{a A}$ & 2,29 cdefA & - \\
\hline RB867515 & $1,01 \mathbf{a b c C}$ & 13,83 bcdef $A$ & $1,36 \mathbf{a b B}$ & $14,96 \mathbf{a b c A}$ & $2,19 \mathbf{a b A}$ & $15,47 \mathbf{a b A}$ & $1,45 \mathbf{a b B}$ & $13,30 \mathbf{a A}$ & $2,64 \mathbf{a b A}$ & - \\
\hline RB92579 & $0,76 \mathrm{Dc}$ & 12,67 defB & $0,92 \mathrm{eB}$ & $12,95 \mathbf{c B}$ & 1,73 defA & $17,79 \mathbf{a b A}$ & 1,21 cdeB & $14,91 \mathrm{aAB}$ & $2,49 \mathbf{a b c A}$ & - \\
\hline RB966928 & $1,01 \mathbf{a b c C}$ & $19,24 \mathbf{a b A}$ & $1,33 \mathbf{a b c B}$ & $20,62 \mathbf{a A}$ & 2,12 abcdA & $17,02 \mathbf{a b A B}$ & $1,40 \mathbf{a b c B}$ & $14,75 \mathrm{aB}$ & 2,43 abcdA & - \\
\hline SP86-0042 & $1,02 \mathbf{a b c C}$ & 15,88 abcdeA & $1,22 \mathbf{a b c d B}$ & $14,44 \mathbf{b c A}$ & 1,93 abcdef $A$ & $18,36 \mathbf{a b A}$ & $1,30 \mathbf{a b c d B}$ & $16,23 \mathbf{a A}$ & 2,35 bcdeA & - \\
\hline CV\% & 9,11 & 8,71 & 7,75 & 15,68 & 4,09 & 11,97 & 6,24 & 11,57 & 5,70 & \\
\hline
\end{tabular}

*Médias seguidas pela mesma letra minúscula na coluna e maiúscula na linha não diferem estatisticamente, pelo teste de Tukey, em nível de 5\% de probabilidade de erro. obs: Para os períodos de 113; 145 e 174 DAP, o comprimento de colmos refere-se à altura média do dossel. Para o período de 203 DAP, refere-se a distância entre o nível do solo até a primeira dewlep visível e, para o período 285, consiste na média de comprimento de 10 colmos (sem o palmito).

Para o número de perfilhos, cinco variedades (CTC2, CTC9, IAC87-3396, IAC91-1099 e RB966928) destacaram-se das demais no início do ciclo (113DAP), porém, no final do ciclo (203DAP), as variedades não mostraram diferenças significativas (Tabela 1). Em suma, as variedades apresentaram maior perfilhamento no início do ciclo (145 e 174 DAP), apresentando uma redução aos 203 DAP. Alguns autores (SILVA et al., 2007) ressalvam que a redução no número de perfilhos é uma característica fisiológica da cultura, a qual pode ocorrer devido à competição por fatores abióticos: luz, água, nutrientes e radiação solar; acarretando minimização dos processos e morte dos perfilhos jovens (OLIVEIRA et al., 2004). BENNET et al. (2011) citam que o perfilhamento está ligado ao potencial genético de cada variedade, sendo fator fundamental na produção da cana-de-açúcar, juntamente com a altura e o diâmetro de colmos. Reduções nas taxas de perfilhamento podem ser encontradas na literatura, obtendo-se reduções de $20 \%$ para a variedade RB-867515 (BENNET et al., 2011), 68\% para as variedades IACSP94-2101 e CTC2 $\left(12,16\right.$ perf. $\mathrm{m}^{-1}$ ) e $10 \%$ para a variedade RB867515 (12 perf. $\mathrm{m}^{-1}$ ) (SANTIN et al., 2009, corroborando os resultados encontrados neste trabalho). A variedade CTC18 apresentou a menor média de perfilhamento, em todos os tempos de avaliação (Tabela 1). Resultados semelhantes foram observados para a variedade IACSP962042 (MACHADO et al., 2009) e para o híbrido HV241 de capim-elefante (Pennisetum purpureum Schum) (BARRETO et al., 2001).

O efeito do déficit hídrico sob o perfilhamento de gramíneas é bastante controverso, estando ligado diretamente ao estádio de desenvolvimento da cultura (MACHADO et al., 2009). O bom perfilhamento possibilita a manutenção da população final ao longo dos cortes. Por outro lado, variedades com baixa taxa de perfilhamento acarretam a obrigatoriedade de capinas nas entrelinhas.

Dez variedades (CTC2, CTC9, CTC15, IAC87-3396, IAC91-1099, IACSP93-3096, IACSP94-2101, IACSP95-5000, RB86-7515 e RB92579) obtiveram médias superiores, aos 285 DAP, em relação às demais variedades, no entanto não mostraram diferenças significativas entre si em diâmetro de colmo (Tabela 2). O diâmetro de colmos é dependente de características ligadas ao genótipo (SILVA \& COSTA, 2004), à adubação (BENNET et al., 2011), ao ciclo de maturação (SOARES et al., 2004), ao suprimento hídrico (SILVA et al., 2008) e tem uma relação direta com a produtividade (CARVALHO et al., 2011). 


\section{Produtividade de biomassa}

As variedades CTC2, CTC9, CTC11, CTC15, IAC87-3396, IAC91-1099, RB867515, RB92579 e SP86-0042 obtiveram as maiores médias de produtividade, porém sem diferença significativa entre si. Entre as 16 variedades avaliadas, as produtividades variaram entre 108,6 e $170,26 \mathrm{Mg} \mathrm{ha}^{-1}$.

As maiores médias de produtividade das variedades estão associadas ao melhor desempenho destas em termos de massa de 10 colmos e ao comprimento de colmos, exceto para a variedade CTC2, que apresentou maiores médias de perfilhamento e diâmetro de colmos.

As variedades CTC2, CTC9, IAC87-3396, RB867515, RB92579 e SP86-0042 obtiveram produtividades acima de $140 \mathrm{Mg} \mathrm{ha}^{-1}$, e as variedades CTC15 e IAC91-1099, maiores que $160 \mathrm{Mg}$ ha $^{-1}$ (Tabela 2), tendo, assim, um grande potencial de utilização em condições de ambientes irrigados no Cerrado goiano.

As variedades CTC4, IACSP93-3046, IACSP94-2094, IACSP94-2101, IACSP95-5000 e RB966928 apresentaram produtividade intermediária, entre 130 e $140 \mathrm{Mg} \mathrm{ha}^{-1}$. Mesmo assim, estas produtividades podem ser consideradas expressivas para a região (CONAB, 2012).

A variedade RB96-6928, apesar de apresentar bom perfilhamento e comprimento de colmos, não ficou no grupo de destaque em produtividade, apresentado um desempenho intermediário, com 130,26 Mg ha'-1. A causa disso pode ter sido a obtenção de menores médias de diâmetro de colmo $(27,88 \mathrm{~mm})$.

A variedade CTC18 apresentou a menor produtividade (Tabela 2). A condição de déficit hídrico impactou negativamente o perfilhamento e o acúmulo de biomassa de colmo. O estresse por deficiência hídrica é considerado como o fator de maior restrição na produção e na estabilidade da produtividade de culturas em muitas regiões do mundo (HEINEMANN, 2010). O não atendimento à necessidade evapotranspirométrica durante as etapas fenológicas afeta negativamente a produtividade de várias culturas.

A tolerância ao estresse hídrico está relacionada a fatores morfológicos, fisiológicos, bioquímicos e metabólicos (LARCHER, 2000). Cutículas mais espessas evitam a perda de água por transpiração (CASTRO et al., 2009). Aumentos na síntese de prolina, superóxido dismutase, catalase, peroxidase e ácido abscísico atuam como mensageiros em vias de respostas na percepção e na atuação em vias de crescimento, em situações de estresse hídrico e outros estressores ambientais (SHARMA et al., 2011). GUIMARÃES et al. (2008) verificaram que a variedade RB72454, sob condição de restrição hídrica, obteve maiores teores de prolina associados ao maior acúmulo de massa seca e crescimento de colmos. OLIVEIRA et al. (2011) verificaram que as variedades RB763710, IACSP813250, RB92579, RB72454, RB867515, IACSP801816 e RB85-5453 apresentaram maiores produtividades em sistemas irrigados.

Relações entre diâmetro, altura e massa de colmos é um indicativo prático interessante, pois maiores alturas e rendimentos de biomassa estão associados a menores diâmetros de colmos (DIAS et al., 2012). Tal comportamento não ocorreu para as variedades CTC9, CTC15 e RB86-7515, que apresentaram maiores valores altos de comprimento de colmos (2,71;2,49 e 2,64 m), diâmetro $(28,9 ; 29,5$ e $31,9 \mathrm{~mm})$ e massa de colmos (18,5; 16,3 e $21,0 \mathrm{~kg}$, respectivamente). Isto mostra o efeito que o estresse hídrico pode proporcionar no crescimento da planta, principalmente para variedades mais sensíveis.

\section{Análise tecnológica}

As variedades apresentaram diferenças significativas para todas as variáveis (TPH, ATR, POL e Umidade), exceto para a variável PUREZA (Tabela 2). Os maiores valores de TPH, ATR e POL foram observados nas variedades CTC9, CTC11, IAC87-3396, IAC91-1099 e SP86-0042. Ainda, a variedade CTC9 apresentou um menor valor de umidade, mais próximo do recomendado (70\%), 
facilitando a queima do bagaço nas caldeiras, no processo de geração de energia (MARQUES et al., 2008).

Os valores encontrados de ATR neste trabalho variaram de 106,14 a 146,12 $\mathrm{kg}$ entre as variedades. Estes valores são similares aos encontrados por VIEIRA et al. (2012), os quais variaram na ordem de 110 a $147 \mathrm{~kg}$ de ATR.

A média geral de fibra foi de 11,27\%, valor similar ao obtido por MASCHIO (2011), de 11,3\% para variedades com irrigação suplementar de 70\% do déficit hídrico. As variedades CTC2, CTC9, CTC15 e RB966928 ficaram, respectivamente, 18\%, 13\%, 3\% e 19,6\% superiores em termos de fibra aos valores encontrados por MASCHIO(2011). Este fato pode ser explicado pelo déficit hídrico mais acentuado no trabalho conduzido com irrigação suplementar de $50 \%$ do déficit. Estes teores de fibras ainda são considerados baixos para a sustentação da cana-de-açúcar em altas produtividades, que devem ter em média $12,5 \%$, pois constata-se em campo que, devido à grande quantidade de massa verde, podem acamar, ocasionando perdas durante o processo de colheita mecanizada (MARQUES et al., 2008).

As maiores (23,19 $\left.\mathrm{t} \mathrm{ha}^{-1}\right)$ e menores $\left(12,86 \mathrm{t} \mathrm{ha}^{-1}\right)$ médias de TPH foram observadas nas variedades CTC9 e CTC18, respectivamente (Tabela 2). Altos valores de TPH (maior que $20 \mathrm{Mg}$ $\mathrm{ha}^{-1}$ ) mostram uma grande viabilidade econômica no processamento industrial (LANDELL \& BRESSIANI, 2008). Apesar de algumas variedades apresentarem valores de TPH altos, estas ficaram limitadas à época de colheita, sendo que poderiam ter alcançado valores superiores por se tratar de variedades com ciclo médio ou médio-tardio.

Para a variável POL, os valores obtidos variaram de 9,40 a 14,66\%, entre as 16 variedades avaliadas. Entretanto, diferenças significativas foram observadas apenas entre a variável CTC9, que obteve o maior valor numérico, e as variedades CTC4, CTC15, CTC18, IACSP94-2101, RB867515 e RB92579. Ainda, as variedades CTC4, RB86-7515 e RB92579 também obtiveram os piores resultados para a variável Pureza (Tabela 2).

A variedade RB92579 apresentou uma matéria-prima de baixa qualidade, devido aos baixos valores de ATR, POL, Pureza e alta Umidade. GUIMARÃES et al. (2011) afirmam que o teor de sacarose (POL) desta variedade é influenciado negativamente por condições de déficit hídrico, o que pode ter contribuído para o baixo desempenho. MASCHIO (2011) observou que esta mesma variedade em condições de estresse hídrico de 70\% obteve um declínio acentuado na produtividade. 
TABELA 2. Massa de 10 colmos (kg) e diâmetro médio de colmos (mm) aos 203 DAP. Produtividade e análise tecnológica da cana-de-açúcar no momento da colheita, aos 396 DAP. Weight (kg) and diameter (mm) of 10 stems to 203 DAP (Day after planting). Yield and technologic analysis of sugar cane in harvest time to 396 DAP.

\begin{tabular}{|c|c|c|c|c|c|c|c|c|c|}
\hline Tratamentos & $\begin{array}{l}\text { Massa de } 10 \\
\text { colmos }\end{array}$ & $\begin{array}{l}\text { Diâmetro médio } \\
\text { de colmos }\end{array}$ & Produtividade & TPH & ATR & POL & Pureza & Fibra & Umidade \\
\hline & $(\mathrm{kg})$ & $(\mathrm{mm})$ & $\left(\mathrm{Mg} \mathrm{ha}^{-1}\right)$ & $\left(\mathrm{t} \mathrm{ha}^{-1}\right)$ & $(\mathrm{kg})$ & $\%$ & $\%$ & $\%$ & $\%$ \\
\hline$\overline{\mathrm{CTC} 2}$ & $14,40 \mathbf{b c d}^{1}$ & 28,60 abcdef & 151,93 abc & 17,72 bcde & $122,90 \mathbf{a b c}$ & $11,69 \mathbf{b c}$ & 80,55 abc & $11,98 \mathbf{a}$ & $72,93 \mathbf{a b}$ \\
\hline CTC4 & $14,00 \mathbf{b c d}$ & 27,13 ef & $140,68 \mathbf{c d}$ & $15,34 \mathbf{e f}$ & $118,35 \mathbf{b c}$ & $10,94 \mathbf{b c}$ & $77,72 \mathbf{b c}$ & $10,49 \mathbf{a}$ & $75,09 \mathbf{a}$ \\
\hline CTC9 & $18,50 \mathbf{a b}$ & 28,90 abcdef & $157,48 \mathbf{a b c}$ & $23,19^{\mathrm{a}}$ & $146,12 \mathbf{a}$ & $14,66 \mathbf{a}$ & $86,89 \mathbf{a}$ & $11,90 \mathbf{a}$ & $70,56 \mathbf{b}$ \\
\hline CTC11 & $15,10 \mathbf{b c}$ & 28,25 cdef & $162,62 \mathbf{a b}$ & $20,90 \mathbf{a b}$ & $131,78 \mathbf{a b}$ & $12,84 \mathbf{a b}$ & $83,26 \mathbf{a b}$ & $11,17 \mathbf{a}$ & $72,87 \mathbf{a b}$ \\
\hline CTC15 & $16,30 \mathbf{a b c}$ & 29,53 abcde & $170,26 \mathbf{a}$ & 20,03 abcd & $124,50 \mathbf{b c}$ & $11,82 \mathbf{b c}$ & $80,04 \mathbf{a b c}$ & $12,32 \mathbf{a}$ & $72,36 \mathbf{a b}$ \\
\hline CTC18 & $12,95 \mathbf{c d}$ & 28,40 bcdef & $108,60 \mathbf{e}$ & $12,86 \mathbf{f}$ & $123,59 \mathbf{b c}$ & $11,85 \mathbf{b c}$ & $81,67 \mathbf{a b}$ & $11,38 \mathbf{a}$ & $73,59 \mathbf{a b}$ \\
\hline IAC87-3396 & $16,45 \mathbf{a b c}$ & 30,38 abcde & $154,85 \mathbf{a b c}$ & 18,90 abcde & $127,12 \mathbf{a b}$ & $12,21 \mathbf{a b}$ & $81,47 \mathbf{a b c}$ & $11,79 \mathbf{a}$ & $72,64 \mathbf{a b}$ \\
\hline IAC $91-1099$ & $15,40 \mathbf{b c}$ & $32,15 \mathbf{a}$ & $168,59 \mathbf{a}$ & $20,67 \mathbf{a b c}$ & $127,38 \mathbf{a b}$ & $12,26 \mathbf{a b}$ & $81,99 \mathbf{a b}$ & $10,67 \mathbf{a}$ & $73,90 \mathbf{a b}$ \\
\hline IACSP93-3096 & $15,10 \mathbf{b c}$ & $31,65 \mathbf{a b c}$ & $137,63 \mathbf{c d}$ & 16,98 bcdef & $128,24 \mathbf{a b}$ & $12,36 \mathbf{a b}$ & $82,20 \mathbf{a b}$ & $10,27 \mathbf{a}$ & $74,26 \mathbf{a b}$ \\
\hline IACSP94-2094 & $10,55 \mathbf{d}$ & $25,23 \mathbf{f}$ & $130,82 \mathbf{d}$ & 16,74 bcdef & $131,46 \mathbf{a b}$ & $12,84 \mathbf{a b}$ & $83,47 \mathbf{a b}$ & $11,94 \mathbf{a}$ & $72,10 \mathbf{a b}$ \\
\hline IACSP94-2101 & $14,65 \mathbf{b c d}$ & 29,68 abcde & $129,99 \mathbf{d}$ & 15,33 def & $123,20 \mathbf{b c}$ & $11,71 \mathbf{b c}$ & $80,58 \mathbf{a b c}$ & $10,87 \mathbf{a}$ & $74,14 \mathbf{a b}$ \\
\hline IACSP95-5000 & $15,80 \mathbf{b c}$ & 31,35 abcd & $144,57 \mathbf{b c d}$ & 18,28 bcde & $130,84 \mathbf{a b}$ & $12,65 \mathbf{a b}$ & $82,19 \mathbf{a b}$ & $11,22 \mathbf{a}$ & $72,85 \mathbf{a b}$ \\
\hline RB867515 & $21,05 \mathbf{a}$ & $31,98 \mathbf{a b}$ & $154,98 \mathbf{a b c}$ & 17,69 bcde & $121,15 \mathbf{b c}$ & $11,41 \mathbf{b c}$ & $79,59 \mathbf{b c}$ & $10,97 \mathbf{a}$ & $74,21 \mathbf{a b}$ \\
\hline RB92579 & $18,30 \mathbf{a b}$ & 30,58abcde & $156,93 \mathbf{a b c}$ & 15,02 ef & $106,14 \mathrm{c}$ & $9,40 \mathbf{c}$ & $74,39 \mathrm{c}$ & $11,00 \mathbf{a}$ & $75,96 \mathbf{a}$ \\
\hline RB966928 & $15,90 \mathbf{a b c}$ & 27,88 def & $130,26 \mathbf{d}$ & 16,12 cdef & $127,51 \mathbf{a b}$ & $12,33 \mathbf{a b}$ & $82,42 \mathbf{a b}$ & $11,72 \mathbf{a}$ & $72,76 \mathbf{a b}$ \\
\hline SP86-0042 & $14,20 \mathbf{b c d}$ & 27,43 ef & $156,93 \mathbf{a b c}$ & 19,18 abcde & $127,72 \mathbf{a b}$ & $12,29 \mathbf{a b}$ & $81,97 \mathbf{a b}$ & $10,61 \mathbf{a}$ & $73,92 \mathbf{a b}$ \\
\hline $\mathrm{CV}$ & $6,04 \%$ & $4,94 \%$ & $5,58 \%$ & $10,4 \%$ & $6,27 \%$ & $8,90 \%$ & $3,48 \%$ & $9,06 \%$ & $2,39 \%$ \\
\hline
\end{tabular}

${ }^{1}$ Médias seguidas da mesma letra na coluna não diferem entre si, pelo teste de Tukey, ao nível de 5\% de probabilidade de erro.

\section{CONCLUSÕES}

Considerando as 16 variedades estudadas nas condições do experimento, concluiu-se que a irrigação suplementar da cana-de-açúcar no cerrado mostrou-se, em geral, altamente viável com ampla resposta à maioria das variedades, obtendo-se a produtividade média de colmos de $147 \mathrm{Mg}$ ha $^{-1}$ (correspondente em pol a 17,8 $\mathrm{Mg} \mathrm{ha}^{-1}$ ), sendo oito delas acima desse valor, de forma a permitir um amplo manejo varietal sob irrigação.

\section{REFERÊNCIAS}

AGÊNCIA NACIONAL DE ÁGUAS. Conjuntura dos recursos hídricos no Brasil: informe 2012. Brasília: ANA, 2012. 215 p.

BARRETO, G. P.; LIRA, M. A.; SANTOS, M. V. F.; DUBEUX JUNIOR, J. C. B. Avaliação de clones de capim-elefante (Pennisetum purpureum Schum.) e de um híbrido com o milheto (Pennisetum glaucum (L.) R. Br.) submetidos a estresse hídrico. 1. Parâmetros morfológicos. Revista Brasileira de Zootecnia, Viçosa, MG, v.30, n.1, p.1-6, 2001.

BENETT, C. G. S.; BUZETTI, S.; SILVA, K. S.; TEIXEIRA FILHO, M. C. M.; GARCIA, C. M. DE P.; MAESTRELO, P. R. Produtividade e desenvolvimento da cana-planta e soca em função de doses e fontes de manganês. Revista Brasileira de Ciência do Solo, Viçosa, MG, v. 35, n.5, p.16611667, 2011.

CAMPOS, C. M.; MILAN, M.; SIQUEIRA, L. F. F. Identificação e avaliação de variáveis críticas no processo de produção da cana-de-açúcar. Engenharia Agrícola, Jaboticabal, v.28, n.3, p.554-564, 2008.

CANASAT. Monitoramento de cana-de-açúcar via imagens de satélite. Disponível em: <http://www.dsr.inpe.br/laf/canasat/>. Acesso em: 1 abr. 2013.

CASTRO, E. M.; PEREIRA, F. J.; PAIVA, R. Histologia vegetal: estrutura e função de órgãos vegetativos. Lavras: UFLA, 2009. 234 p.

CARVALHO, L. A.; SILVA JUNIOR, C. A.; NUNES, W. A. G. A.; MEURER, I.; SOUZA JÚNIOR, W. S. Produtividade e viabilidade econômica da cana-de-açúcar em diferentes sistemas de 
preparo do solo no centro-oeste do Brasil. Revista de Ciências Agrárias. Lisboa, v.34, n.1, p. 199211. 2011.

CONAB. Companhia Nacional de Abastecimento. Acompanhamento de safra brasileira: cana-deaçúcar, terceiro levantamento, dezembro/2012. Brasília, DF, Conab 2013. 18 p.

DALRI, A. B.; CRUZ, R. L. Produtividade da cana-de-açúcar fertirrigada com N e K via gotejamento subsuperficial. Engenharia Agrícola, Jaboticabal, v.28, n. 3, p. 516-524, 2008.

DIAS, C. M. O.; CORSATO, C. E.; SANTOS, V. M.; SANTOS, A. F. S. Indicadores fitotécnicos, de produção e agroindustriais em cana-de-açúcar cultivada sob dois regimes hídricos. Revista Caatinga, Mossoró, v. 25, n. 3, p. 58-65, 2012.

EMBRAPA-Centro Nacional de Pesquisa de Solos. Sistema brasileiro de classificação de solos. 2. ed. Rio de Janeiro: EMBRAPA-SPI, 2006. 306 p.

FARIAS, C. H. A.; FERNANDES, P. D.; DANTAS NETO, J.; GHEYI, H. R. Eficiência no uso da água na cana-de-açúcar sob diferentes lâminas de irrigação e níveis de zinco no litoral paraibano. Engenharia Agrícola, Jaboticabal, v.28, n.3, p. 494-506, 2008.

GONÇALVES, E. R.; FERREIRA, V. M.; SILVA, J. V.; ENDRES, L. B.; TADEU, P.; DUARTE, W. G. Trocas gasosas e fluorescência da clorofila a em variedades de cana-de-açúcar submetidas à deficiência hídrica. Revista Brasileira de Engenharia Agrícola e Ambiental, Campina Grande, v.14, n.4, p.378-386, 2010.

GUIMARAES, E. R.; MUTTON, M. A.; MUTTON, M. J. R.; FERRO, M. I. T.; RAVANELI, G. C.; SILVA, J. A. Free proline accumulation in sugarcane under water restriction and spittlebug infestation. Scientia Agricola, Piracicaba, v.65, n.6, p.628-633, 2008.

HENRY, R. J. Basic Information on the sugarcane plant.In: HENRY, R.; KOLE, C. (Ed.). Genetics, genomics and breeding of sugarcane. New York: CRC Press, 2010. p.1-7.

LANDELL, M. G. A.; SILVA, M. A. As estratégias de seleção da cana em desenvolvimento no Brasil. Visão Agrícola, Piracicaba, v.1, n.1, p. 18-23, 2004.

LANDELL, M.G.A.; BRESSIANI, J.A. Melhoramento genético, caracterização e manejo varietal. In: DINARDO-MIRANDA, L.L.; VASCONCELOS, A.C.M.; LANDELL, M.G.A.(Ed.). Cana-deaçúcar. Campinas: Instituto Agronômico, 2008. p. 101-155.

LARCHER, W. Ecofisiologia vegetal. São Carlos: Rima, 2000. 531p.

LEAL, D. P.V. Evapotranspiração da cana-de-açúcar e fotossíntese acumulada em biomassa e energia, para diferentes variedades, disponibilidades hídricas no solo e ciclos de cultivos. 2012. Dissertação (Mestrado em Engenharia de Sistemas Agrícolas) - Escola Superior de Agricultura Luiz de Queiroz, Universidade de São Paulo, Piracicaba, 2012.

MACHADO, R. S.; RIBEIRO, R. V.; MARCHIORI, P. E. R.; MACHADO, D. F. S. P.; MACHADO, E. C.; LANDELL, M. G. A. Respostas biométricas e fisiológicas ao déficit hídrico em cana-de-açúcar em diferentes fases fenológicas. Pesquisa Agropecuária Brasileira, Brasília, v.44, n.12, p.1575-1582, 2009.

MARIN, F. R.; NASSIF, D. S. P. Mudanças climáticas e a cana-de-açúcar no Brasil: Fisiologia, conjuntura e cenário futuro. Revista Brasileira de Engenharia Agrícola e Ambiental, Campina Grande, v.17, n.2, p.232-239, 2013.

MASCHIO, R. Produtividade da água em biomassa e energia para 24 variedades de cana-deaçúcar. 2011. 87 f. Tese (Mestrado em Ciências: Irrigação e Drenagem)-Escola Superior de Agricultura Luiz de Queiroz, Universidade do Estado de São Paulo, Piracicaba, 2011.

MARQUES, M.O.; MACIEL, B.F.; FIGUEIREDO, I.C.; MARQUES, T.A. Considerações sobre a qualidade da matéria-prima. In: MARQUES M.O., MUTTON, M.A., NOGUEIRA, T.A.R., TASSO 
JÚNIOR, L.C., NOGUEIRA, G.A., BERNARDI, J.H. Tecnologias na agroindústria canavieira. Jaboticabal: FCAV, 2008. p.9-16.

OLIVEIRA, F. M.; ASPIAZU, I.; K, M. K.; BORGES, I. D.; PEGORARO, R. F.; VIANNA, E. J. Crescimento e produção de variedades de cana-de-açúcar influenciadas por diferentes adubações e estresse hídrico. Revista Trópica - Ciências Agrárias e Biológicas, São Luís, v.5, n.1, p.56-67, 2011.

OLIVEIRA, R. A.; DAROS, E.; ZAMBON, J. L. C. Crescimento e desenvolvimento de três cultivares de cana-de-açúcar, em cana-planta, no estado do Paraná. Scientia Agrícola, Piracicaba, v.5, n.1/2, p.87-94, 2004.

SANTIN, I.; SILVA NETO, F.; TASSO JUNIOR, L. C.; MARQUES, M. O. Biometria em cultivares tardios em cana-de-açúcar. In: CONGRESSO DE INICIAÇÃO CIENTIFICA DA UNESP, 21, 2009. São José do Rio Preto. Anais... São Paulo: UNESP, 2009. Disponível em: <http://prope.unesp.br/xxi_cic/27_38396660824.pdf> Acesso em: 28 jan. 2013.

SHABANI, A.; SEPASKHAH, A. A.; KAMGAR-HAGHIGHI, A. A. Responses of agronomic components of rapeseed (Brassica napus L.) as influenced by deficit irrigation, water salinity and planting method. International Journal of Plant Production, Gorgan, v. 7, n. 2, p.313-340, 2013.

SHARMA, D. K.; DUBEY, A. K.; SRIVASTAV, M.; SINGH, A. K.; SAIRAM, R. K.; PANDEY, R. N.; DAHUJA, A.; KAUR, C. Effect of putrescine and paclobutrazol on growth, physiochemical parameters, and nutrient acquisition of salt-sensitive citrus rootstock Karnakhatta (Citrus karnaRaf.) under $\mathrm{NaCl}$ stress. Journal of Plant Growth Regulation, NewYork, v.30, n.3, p.301-311, 2011.

SILVA, M. A.; SILVA, J. A. G.; ENCISO, J.; SHARMA, V.; JIFON, J. Yield components as indicators of drought tolerance of sugarcane. Scientia Agricola, Piracicaba, v.65, n.6, p.620-627, 2008.

SILVA, M. A.; GAVA, G. J. C.; CAPUTO, M. M.; PINCELLI, R. P.; JERONIMO, E. M.; CRUZ, J. C. S. Uso de reguladores de crescimento como potencializadores do perfilhamento e da produtividade da cana-soca. Bragantia, Campinas, v.66, n.4, p.545-552, 2007.

VIEIRA, G. H. S.; MANTOVANI, E. C.; SEDIYAMA, G. C.; COSTA, E. L.; DELAZARI, F. T. Produtividade de colmos e rendimento de açúcares da cana-de-açúcar em função de lâminas de água. Irriga, Botucatu, v.17, n.2, p. 234-244, 2012. 